Original Research Article

\title{
Monitoring cutaneous adverse drug reactions in patients on TDF+3TC+EFV: a single centre experience
}

\author{
Nikhil Era $^{1}$, Shatavisa Mukherjee ${ }^{1 *}$, Bibhuti Saha ${ }^{2}$, Santanu Kumar Tripathi ${ }^{1}$
}

\begin{abstract}
${ }^{1}$ Department of Clinical and Experimental Pharmacology, ${ }^{2}$ Department of Tropical Medicine, Calcutta School of Tropical Medicine, Kolkata 700073, West Bengal, India
\end{abstract}

Received: 02 April 2017 Accepted: 28 April 2017

\section{*Correspondence to:}

Ms. Shatavisa Mukherjee, Email: shatavisa100@ gmail.com

Copyright: (C) the author(s), publisher and licensee Medip Academy. This is an openaccess article distributed under the terms of the Creative Commons Attribution NonCommercial License, which permits unrestricted noncommercial use, distribution, and reproduction in any medium, provided the original work is properly cited.

\begin{abstract}
Background: HIV-infected patients initiating antiretroviral therapy may manifest a wide variety of ADRs ranging from trivial manifestation, such as rashes, pigmentation, to severe life-threatening reactions, such as StevenJohnson syndrome, toxic epidermal necrolysis. The present study thus monitored cutaneous adverse drug reactions in patients on first line antiretroviral regimen comprising of tenofovir disoproxil fumerate, lamivudine and efavirenz as a three drug-combination.

Methods: A prospective observational clinical study was carried out for a period of one year among PLHIV receiving TDF+3TC+EFV as first line regimen in the outpatient setting of a nodal ART centre of eastern India.

Results: The major regimen induced dermatological complications presenting in our study set up included rashes, itching, SJS, pigmentation of nails, skin Hyperpigmentation. The morbilliform eruption, often referred to as a maculopapular rash, is the most common type of reaction occurring after treatment initiation.

Conclusions: Adverse drug reactions are one of the most common public health concerns, which influence patients' treatment options along with health care costs.
\end{abstract}

Keywords: Antiretroviral therapy, Cutaneous adverse drug reactions, Nail pigmentations, Skin hyperpigmentations, Steven-Johnson syndrome

\section{INTRODUCTION}

HIV infected patients have a higher risk of developing cutaneous reactions than the general population, irrespective of age. HIV-infected patients initiating antiretroviral therapy (ART) may manifest a wide variety of ADRs ranging from trivial manifestation, such as pigmentation, to severe life-threatening reactions, such as Steven-Johnson syndrome (SJS), toxic epidermal necrolysis (TEN), exfoliative dermatitis and drug hypersensitivity syndrome. Various patient and drugrelated factors contribute to the risk of adverse drug reactions. Drug hypersensitivity in HIV infected patients has been found to be about 100 times more common than in the general populace.
Adverse drug reactions (ADRs) are common and important public health concern, which may even be life-threatening. Their impact is significant in terms of patients' current and future treatment options along with cost and health service resources. The ultimate goal in a pharmacovigilance program is always to discontinue the offending medication if possible. Individuals with cutaneous drug eruptions are often very ill patients taking a large number of medications, many of which are essential for their survival. Knowing the common eruption-inducing medications may help in identifying the offending drug. The decision of whether to continue to administer a drug that is known or assumed to be the cause of a reaction will be influenced by the following four key factors: the severity and probable course of the reaction; the disease for which the drug was prescribed; 
ease or difficulty with which the reaction can be managed; and the availability of chemically unrelated drugs with similar pharmacologic properties. ${ }^{1}$ However, all nonessential medications should be discontinued. Ascertaining the causality of suspected ADRs still remains a challenge in resource-limited settings, mostly due to the unavailability of qualified personnel in health institutions and the inconsistent laboratory support resulting in poor implementation of pharmacovigilance programs. The present study monitored cADRs in patients put on first line ART comprising of tenofovir disoproxil fumerate, lamivudine and efavirenz, in a tertiary care nodal ART centre.

\section{METHODS}

A prospective observational clinical study was carried out for a period of one year among PLHIV receiving $\mathrm{TDF}+3 \mathrm{TC}+\mathrm{EFV}$ as first line regimen in the outpatient setting of a nodal ART centre of eastern India. Institutional ethics committee approval was taken prior to the initiation of the study and written informed consent was obtained from all subjects before their inclusion in the study. All treatment naïve subjects of either sex aged 18 years or above on ART were included in the study. Subjects having treatment modifications due to virological or immunologic failure, pregnant women, lactating mothers, patients having any other co morbidities like psychiatric illness, diabetic mellitus, hypertension, chronic kidney disease, etc were excluded from the study. Data regarding patient demographics and clinical information were collected in a pre-structured proforma. ADR diagnosis was based on patient complaints and/or morphological changes noticed by physicians during routine clinical exam. Adverse event history, medication history and other relevant details were captured in a format as adopted in the Pharmacovigilance Programme of India (PvPI). Causality of ADR was assessed by Naranjo's ADR probability scale. ${ }^{2}$ The severity of each reported ADR was assessed using Hartwig and Siegel Scale. ${ }^{3}$ Descriptive statistical analysis of the obtained data was performed.

\section{RESULTS}

Out of 242 patients who were included in the study, 45 patients reported with one or more cutaneous adverse drug reaction. The various reported cADRs included rashes, itching, nail pigmentation, skin hyperpigmentation and serious conditions like Stevens Johnson Syndrome.

\section{Rashes}

Out of 45 patients reporting with cADRs, 29 Patients presented with mild to moderate rashes which were symptomatically managed. No dechallenge of regimen was required however. Figure 1, represents a 35 year old female presenting with maculopapular rash extending from face, back and upper extremities to trunk; which resolved after one month of symptomatic treatment (emollients, antihistaminics) followed with no other further recurrence.

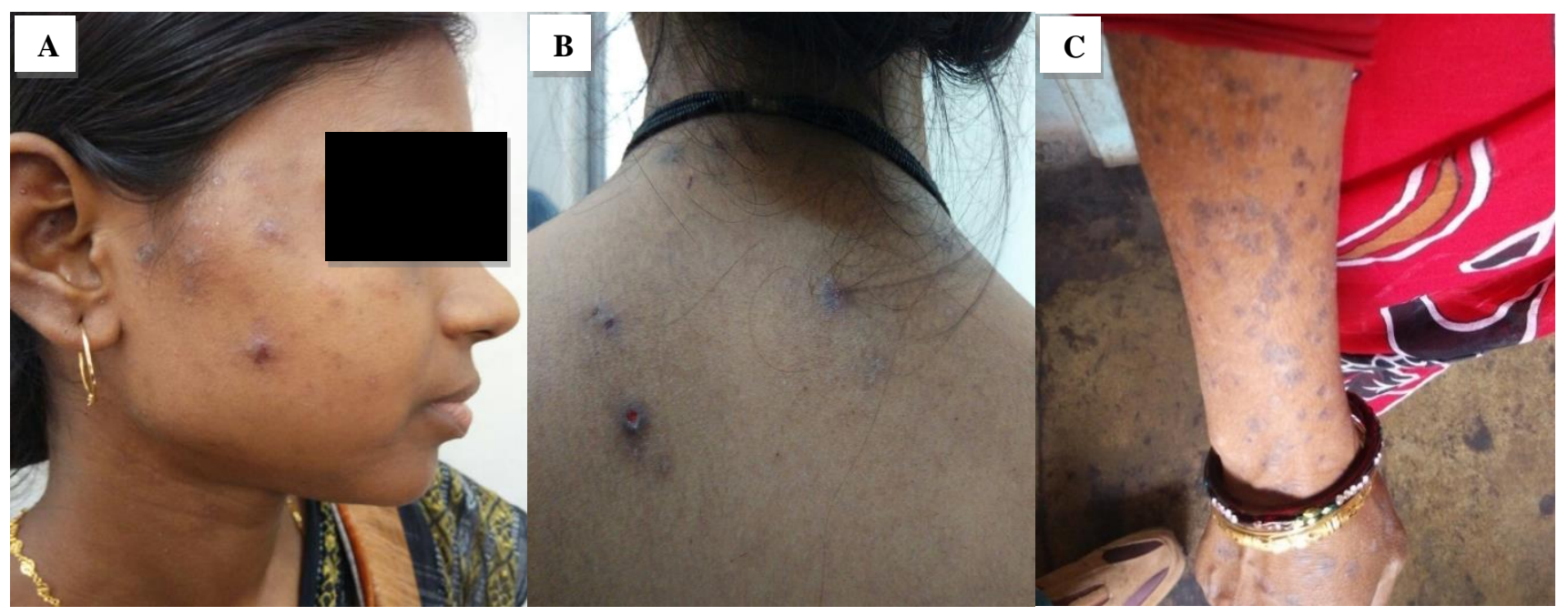

Figure 1 (A, B and C): Rashes in patients on TDF+3TC+EFV.

\section{Nail pigmentations}

5 patients were observed who developed nail pigmentation within 2 to 4 months of regimen initiation.
Presentations mostly involved bluish to brown discolorations of thumbnails and forenails beginning proximally and progressing distally to involve the freeedges of the nails. In some cases the nails were brittle 
with loss of lunulae and periungual changes. No specific treatment was given for nail pigmentation. The patients were counselled about harmless nature of this ADR and continued on the same regimen. Though harmless and reversible, psychological aspects of these side effects may have affected the adherence to the regimen. (Figure 2).

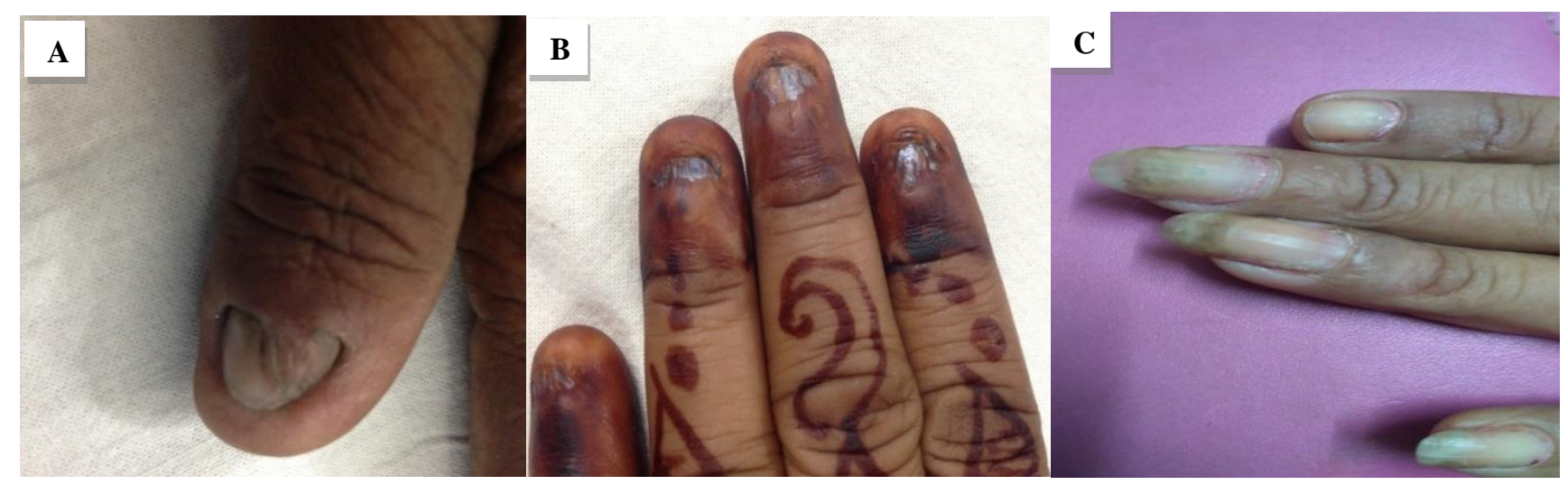

Figure 2 (A, B and C): Nail pigmentations in patients on TDF+3TC+EFV.

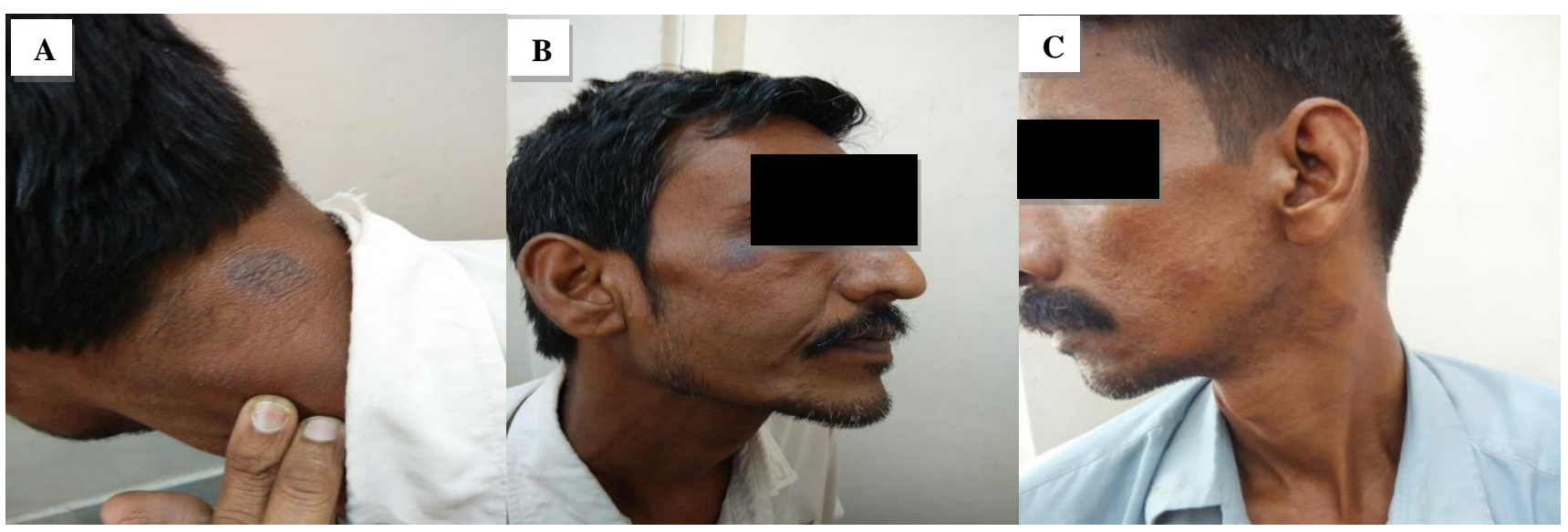

Figure 3 (A, B and C): Skin hyperpigmentations in a patient on TDF+3TC+EFV.

\section{Skin pigmentations}

There were 2 cases of skin pigmentation due this regimen. Figure 3 shows a 49 year old male presenting with bluish grey pigmented macules on sun exposed areas. Another patient presented with similar pigmented macules on extremities and trunk. No discontinuation of regimen was prompted. Both the cases were resolved with two months of its development. Generous use of water based sunscreen was recommended.

In Stevens Johnson Syndrome two such cases were encountered.

\section{Case 1}

A 48 year old male presented with fever and painful generalised maculopapular rash with confluent erythema after 3 weeks of regimen initiation (Figure 4).

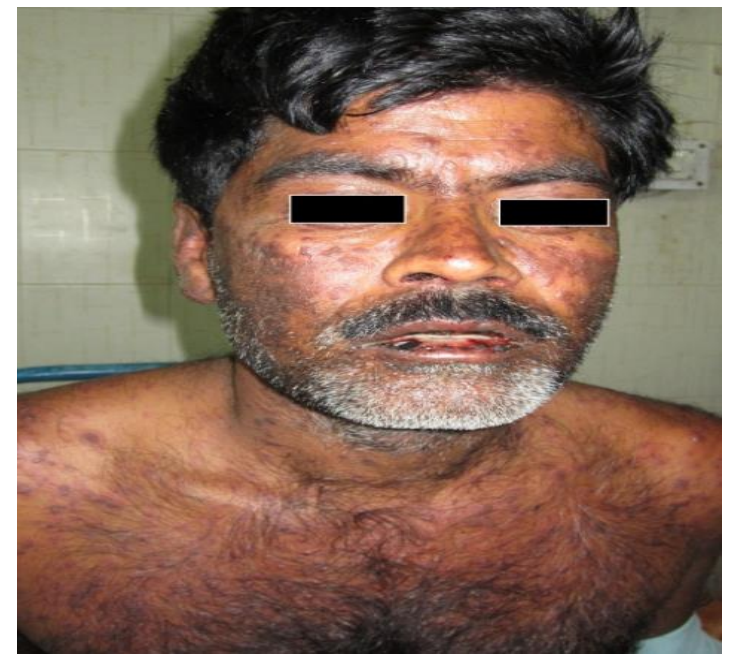

Figure 4: Generalised maculopapular rash and mucositis in 48 year old male. 


\section{Case 2}

A 39 year old female, started on TDF+3TC+EFV developed erythematous maculopapular rash, On oral examination, both upper and lower lips showed swelling extensive irregular ulcerations, showing cracking and fissuring with blood encrustation (Figure 5).

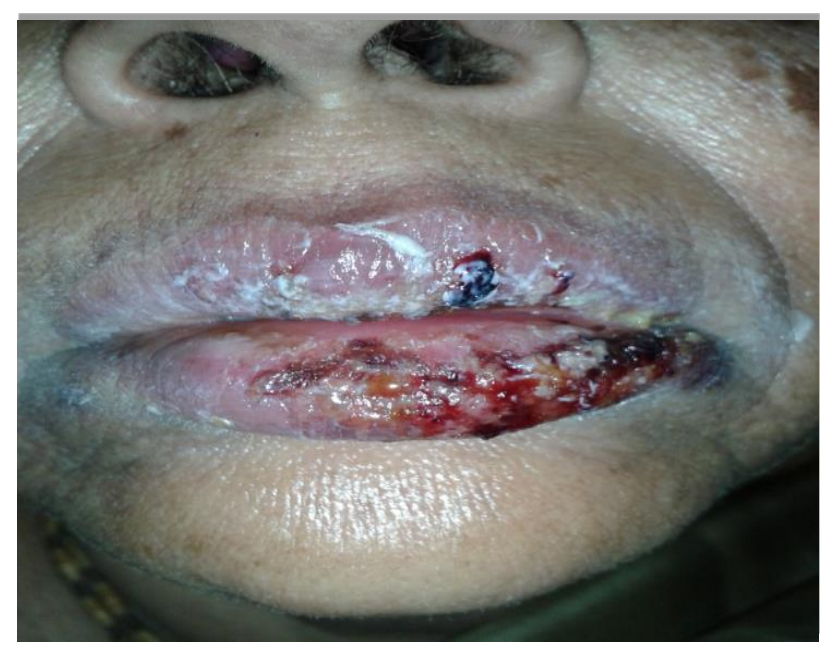

Figure 5: Erythematous purpuric rash with blister formation, lip swelling in 39 year old female.

Both patients were managed in the lines of treatment of Stevens Johnson syndrome (fluid management, antiseptics etc.). The suspected drug in both cases was efavirenz, as dechallenge and successful regimen switch to $\mathrm{TDF}+3 \mathrm{TC}+\mathrm{LPV} / \mathrm{r}$ showed complete remission without any further recurrence of any reactions.

\section{Causality and severity assessment}

Of the total 45 cases observed, causality assessment was performed using Naranjo's algorithm, which conferred all the cases to be probable. Severity of the reported cADRs were assessed using Hartwig Seigel's Scale, which showed 43 cases to be mild, while 2 cases to be severe.

\section{DISCUSSION}

Worldwide statistics states that an estimated 36.7 million people are living with human immunodeficiency virus (PLHIV), and around $46 \%$ of PLHIV were having access to ART globally. ${ }^{4}$ The introduction of highly active antiretroviral therapy (HAART) have brought a ray of hope to PLHIV as it led to significant reduction in acquired immune deficiency syndrome (AIDS)-related morbidity and mortality. The overall benefits of viral suppression and improved immune function as a result of effective ART far outweigh the risks associated with the adverse effects of some antiretroviral (ARV) drugs. Rates of virological failure during first line regimens are decreasing both in clinical trials and in studies performed during routine clinical practice. However, drug-related events like cADRs are increasingly recognized and represent one of the most common reasons for treatment discontinuation or switch. As ART is now recommended for all patients regardless of CD4 T lymphocyte (CD4) cell count, and therapy has to be continued indefinitely, the focus of patient management has evolved from identifying and managing early ARV-related toxicities to individualizing therapy to avoid long-term adverse effects. ${ }^{5}$ According to Holly et al, a patient must be initiated on antiretroviral (ARV) drugs with nonoverlapping toxicities and a small risk of interaction with other existing treatments. ${ }^{6}$ Post adverse reaction rechallenge should be medically supervised. The present study probed into the cutaneous adverse effects of the first line regimen consisting of tenofovir, lamivudine and efavirenz.

Cutaneous adverse drug reactions have been reported with all antiretroviral medications. HIV-infected patients initiated on ART can frequently show a wide variety of adverse drug effects such as drug rashes, hyperpigmentation, hair loss, hypersensitivity reactions, injection site reaction, urticarial reaction, erythema multiforme, toxic epidermal necrolysis (TEN) or Stevens-Johnson syndrome (SJS). Therefore, it is necessary to develop and get approval of novel antiretrovirals as soon as possible in order to avoid these cutaneous adverse reactions. ${ }^{7}$ Our study showed that the major regimen induced dermatological complications presenting in our study set up included rashes, itching, SJS, pigmentation of nails, skin hyper pigmentation respectively. The morbilliform eruption, often referred to as a maculopapular rash, is the most common type of reaction after treatment. Nail and skin hyperpigmentation have been reported in long-standing patients infected with HIV.

Hyperpigmentation has been observed as a manifestation of photosensitivity in HIV-infected patients on first line regimens. These adverse effects resemble the dermatological effects of retinoids. Homologies between the amino acid sequences of retinoic acid-binding protein 1 and the catalytic site of HIV type-1 (HIV-1) proteases have been noted. Hyperpigmentations due to melanin incorporation are occasionally observed during antiretroviral therapy, most commonly as longitudinal, and more rarely as mucosal hyperpigmentation. The longitudinal discoloration of the nails is diagnosed especially during therapy with $\mathrm{TDF} / \mathrm{EFV}$ more rarely with lamivudine (3TC), with an increased incidence and intensity in the dark skin type. The hyperpigmentation may fade after switching medications.

Moreover, drug induced nail pigmentation typically involves several nails and is usually reversible. However, it may take months to several years to recover melanin production by melanocytes of the nail matrix after drug withdrawal. ${ }^{8}$

Drug hypersensitivity in HIV infected patients typically manifests as erythematous maculopapular, pruritic and 
confluent rash with or without fever. Rash is most prominent on the body and arms and begins after one to two weeks of therapy. While therapy may sometimes be continued in the presence of maculopapular exanthem, it must be discontinued in cases of serious adverse drug reactions. These are classified into Stevens-Johnson syndrome (SJS), SJS/TEN overlap and toxic epidermal necrolysis (TEN). Multiform erythemas develop on the integument, which usually do not exhibit the concentric annular form of erythema exsudativum multiforme (EEM). Erosions develop on the mucous membranes, at the body orifices with crusty deposits or whitish pseudo membranes. Involvement of the oral mucosa is typical. ${ }^{8}$ In the SJS/TEN transitional form, $10 \%$ to $30 \%$ of the skin is affected and in TEN more than $30 \%$ of the skin. Treatment mainly consists of medication discontinuation and is in the line of management of severe burn. According to Carr et al., Stevens Johnson syndrome or toxic epidermal necrolysis have been found to develop in less than $0.3 \%$ of patients on highly active antiretroviral therapy (HAART). ${ }^{9}$

Our study had certain limitations also. Being an OPD based study, it is quite possible that some ADRs were missed that were transient or too mild to have inconvenienced the patient to report. Being a government set up, no detailed investigations could be ordered apart from routine laboratory investigations. Moreover the study was conducted for a short period at a single centre with a small sample size, thus the data cannot be a representative of national statistics. The study failed to identify the potential predictors of ADRs to ART in HIV infected patients. Risk factor correlation was not studied. Thus, presence of other confounding factors which could have affected the final outcome of the study which were beyond the scope of current study remains a faint possibility. Moreover, the study time period was much shorter for adjudging long term complications of this regimen. Thus only a trend towards aforesaid complications could be determined.

\section{CONCLUSION}

Various reactions ranging from mild rashes to severe SJS were encountered in patients initiated on TDF+3TC+EFV. Discontinuation or switch remains the common options in severe forms of cADRs, which ignites the need of active pharmacovigilance programme as ADRs significantly influences patients' current and future treatment options along with cost and available health service resources.
Funding: No funding sources Conflict of interest: None declared

Ethical approval: The study was approved by the Institutional Ethics Committee

\section{REFERENCES}

1. Mudzviti T, Sibanda M, Gavi S, Maponga CC, Morse GD. Implementing a pharmacovigilance program to evaluate cutaneous adverse drug reactions in an antiretroviral access program. Journal of infection in developing countries. 2012;6(11):806-8.

2. Naranjo CA, Busto U, Sellers EM. A method for estimating the probability of adverse drug reactions. Clin Pharmacol Ther. 1981 Aug; 30(2):239-45.

3. Hartwig SC, Siegel J, Schneider PJ. Preventability and severity assessment in reporting adverse drug reactions. Am J Hosp Pharm. 1992 Sep; 49(9):222932.

4. HIV/AIDS. World Health Organization. Available from: http://www.who.int/gho/hiv/en/. Accessed on April 01, 2017

5. Adverse effects of ARV. Available from: https://aidsinfo.nih.gov/guidelines/html/1/adult-andadolescent-arv-guidelines/31/adverse-effects-of-arv Accessed on April 01, 2017

6. Shear NH, Milpied, Bruynzeel DP, Phillips EJ. A review of drug patch testing and implications for HIV clinician. AIDS. 2008;22:999-1007.

7. Borras-Blasco J, Navarro-Ruiz A. Adverse cutaneous reactions as associated with the newest antiretroviral drugs in patients with human immunodeficiency virus infection. Journal of antimicrobial chemotherapy. 2008;62:879-888.

8. Hartmann M, Enk A. Cutaneous Effects of Antiretroviral Therapy. Dtsch Arztebl. 2007;104(16):A 1098-1103.

9. Carr A, Vella S, de Jong MD, Sorice F, Imrie A, Boucher CA, et al. controlled trial of nevirapine plus zidovudine versus zidovudine alone in p24 antigenaemic HIV-infected patients. The DutchItalian - Australian Nevirapine Study Group. AIDS. 1996;10:635-41.

Cite this article as: Era N, Mukherjee S, Saha B, Tripathi SK. Monitoring cutaneous adverse drug reactions in patients on $\mathrm{TDF}+3 \mathrm{TC}+\mathrm{EFV}$ : a single centre experience. Int J Basic Clin Pharmacol 2017;6:1467-71. 